\title{
Prevalência dos fatores de risco da doença coronariana em paciente submetidos a revascularização do miocárdio
}

\author{
Prevalence of coronary heart disease risk factors in patients undergoing coronary artery \\ surgery
}
Prevalencia de factores de riesgo de enfermedad coronária em pacientes sometidos a cirugía de artéria coronaria

Mariana Miqueletti Gomes Tavares ${ }^{1 *}$, Gérlia Bernardes da Silveira1, Gabriel Valentim de Castro, Sarah da Silva Candido², Cynthia Antônia Kallás Bachur ${ }^{1,2}$.

\section{RESUMO}

Objetivo: Identificar os fatores de risco prevalentes para o desenvolvimento de doenças cardiovasculares em pacientes submetidos a cirurgia de revascularização miocárdica. Métodos: Trata-se de um estudo observacional, analítico, retrospectivo, com levantamento dos fatores de risco para o desenvolvimento de doença arterial crônica, a partir da análise de prontuários de pacientes submetidos a cirurgia de revascularização miocárdica, no período de 2010 a 2016. Resultados: Foram selecionados 206 prontuários, sendo $60(29,1 \%)$ do sexo feminino e $146(70,8 \%)$ do sexo masculino, com idade média de $65,5 \pm 8,45$ e 63,5 $\pm 9,16$, respectivamente. Os principais fatores de risco para o desenvolvimento de doenças cardiovasculares identificados foram: classificação do índice de massa corporal, que apresentou grande percentual na categoria "Sobrepeso" 74 (46,8\%), seguido por "Obesidade grau I" 46 (29,1\%) e a Hipertensão arterial presente em 172 $(83,4 \%)$ dos pacientes analisados. Conclusão: Medidas socioeducativas podem ser tomadas ou intensificadas para a prevenção da síndrome coronariana aguda e consequentemente da revascularização miocárdica, como a distribuição dos existentes guias nutricionais, programas de academias públicas, pistas de caminhada e estímulo de práticas saudáveis.

Palavras-chave: Revascularização miocárdica, Fatores de risco, Profissionais de saúde.

\begin{abstract}
Objective: To identify the most prevalent risk factors of the development of cardiovascular diseases in patients undergoing coronary artery bypass grafting at public hospital in a city of interior of state of São Paulo. Methods: This was an observational, analytical, and retrospective study with a survey of risk factors for the development of coronary artery disease, based on the analysis of medical records of patients submitted to myocardial revascularization surgery, from 2010 to 2016. Results: 206 medical records were selected, 60 $(39,1 \%)$ female and $146(70,8 \%)$ male, with a mean age of $65,5 \pm 8,45$ and $63,5 \pm 9,16$ respectively. The main risk factors for the development of cardiovascular diseases identified were: classification of body mass index, which presented a high percentage in the category "Overweight" $74(46,8 \%)$ followed by "Grade I obesity" 46 $(29,1 \%)$, and arterial hypertension present in $172(83,4 \%)$ of the patients analyzed. Conclusion: Socioeducational measures may be taken or intensified for the prevention of acute coronary syndrome and consequently for myocardial revascularization, such as the distribution of existing nutritional guides, public health programs, walking paths and encouragement of healthy practices.
\end{abstract}

Keywords: Myocardial revascularization, Risk factors, Health professionals.

${ }^{1}$ Universidade de Franca (UNIFRAN), Franca - SP. *E-mail: marianamiquelettitavares@hotmail.com

2 Universidade de São Paulo (USP), Ribeirão Preto - SP. 


\section{RESUMEN}

Objetivo: Identificar los factores de riesgo más prevalentes del desarrollo de enfermedades cardiovasculares en pacientes sometidos a injerto de revascularización coronaria en un hospital público de una ciudad del interior del estado de São Paulo. Métodos: Estudio observacional, analítico y retrospectivo con una encuesta de factores de riesgo para el desarrollo de enfermedad coronaria, basado en el análisis de los registros médicos de pacientes sometidos a cirugía de revascularización miocárdica, de 2010 a 2016. Resultados: Fueron seleccionados 206 registros, $60(39,1 \%)$ mujeres y $146(70,8 \%)$ hombres, con una edad media de $65,5 \pm 8,45$ y $63,5 \pm 9,16$ respectivamente. Los principales factores de riesgo para el desarrollo de enfermedades cardiovasculares identificadas fueron: clasificación del índice de masa corporal, que presentó un alto porcentaje en la categoría "Sobrepeso" 74 (46,8\%), seguido de "Obesidad Grado I" 46 (29,1\%) e hipertensión arterial presente en $172(83,4 \%)$ de los pacientes analizados. Conclusión: Se pueden tomar o intensificar medidas socioeducativas para la prevención del síndrome coronario agudo y, en consecuencia, para la revascularización miocárdica, como la distribución de guías nutricionales existentes, programas de salud pública, senderos para caminar y el fomento de prácticas saludables.

Palabras clave: Revascularización miocárdica, Factores de riesgo, Profesionales de la salud.

\section{INTRODUÇÃO}

As doenças cardiovasculares (DCV) constituem quase metade das doenças não transmissíveis, o que pode chegar a mais de 23,6 milhões por volta de 2030. As populações afetadas são aquelas que vivem em países de renda baixa ou média $(80 \%)$, ocorrendo geralmente em indivíduos mais jovens do que aqueles acometidos nos países de renda mais alta.

No Brasil, em 2009, as doenças do aparelho circulatório foram as principais causas de óbitos (29\%) seguidas por neoplasias, causas externas e doenças do aparelho respiratório, que juntas totalizaram $60 \%$ das mortes naquele ano, sendo a faixa etária entre 40 a 59 anos a mais afetada. Já em 2012, no Brasil, as doenças do aparelho circulatório foram a principal causa de óbito em homens, o que responde por $26 \%$ do total das mortes neste ano (FERRERIA AG, et al., 2013).

Segundo a Organização Mundial da Saúde (OMS) as DCV são responsáveis por 30\% do total de mortes no mundo. A doença arterial coronariana (DAC) afeta pessoas de todas as etnias, mas a sua incidência é consideravelmente mais elevada entre os caucasianos. Já entre os negros a taxa de mortalidade é mais elevada até os 60 anos em homens e nas mulheres, até os 75 anos de idade. No entanto, a etnia por si só não parece ser um fator tão importante quando comparada com o hábito de vida do indivíduo.

A mortalidade relacionada à DAC oscila entre 11,3 a 2,5 óbitos por 100.000 brasileiros, o que depende de fatores locais, sendo o Rio de Janeiro o estado que apresenta maior índice de mortalidade, apesar deste índice, nota-se que existe um declínio dessas taxas nos últimos anos (ALVES A e MAQUES IR, 2009).

Cerca de $40 \%$ das DAC têm sua primeira manifestação como infarto agudo do miocárdio (IAM) e de $10 \%$ a $20 \%$ dos casos se apresentam como morte súbita (FERREIRA AG, et al., 2013), que ocorre devido a lesões na camada íntima das coronárias, podendo envolver também a média e a adventícia, que evoluem com a formação de placas ateroscleróticas. Os depósitos de gordura (chamados ateromas ou placas) formam-se paulatinamente e desenvolvem-se, na maioria das vezes, irregularmente nos grandes troncos das duas artérias coronárias principais. Este processo é conhecido como aterosclerose. Uma lesão simples pode acumular, progressivamente, lipídios, plaquetas, fina capa de fibrose e desenvolver uma intensa resposta inflamatória. Promove, também, a inibição da síntese do colágeno pelos linfócitos T e aumento da degradação do colágeno, o que culmina com a ruptura da placa que, por sua vez, pode levar à formação do trombo, manifestada de diversas maneiras (LIMA RC, et al., 2004).

Quando esse processo ocorre em um indivíduo, desenvolve-se a obstrução de uma artéria coronária, que pode culminar em uma isquemia do músculo cardíaco (miocárdio), causando lesões mais graves, uma vez que, para que o coração se contraia e bombeie o sangue normalmente, o miocárdio requer uma perfusão 
contínua de sangue rico em oxigênio que as artérias coronárias lhe proporcionam. A causa mais frequente de isquemia do miocárdio é a DAC. As sérias consequências podem manifestar-se clinicamente como angina estável, angina instável ou IAM (FERNANDES PM, 2008).

Dentre os fatores de risco para DAC, os que mais se destacam são: hereditariedade, sexo masculino, idade avançada, hipertensão arterial sistêmica (HAS), diabetes mellitus (DM), dislipidemia (DLP), obesidade, sedentarismo, tabagismo. Hereditariedade, gênero e idade são denominados fatores de risco constitucionais, ou seja, não modificáveis, sendo os demais comportamentais ou modificáveis (LIMA RC et al., 2004; LIMA FET, et al., 2012).

Sendo assim, a cirurgia de revascularização do miocárdio (RM) é uma das formas de tratamento da insuficiência coronariana, com objetivo de reestabelecer o suprimento sanguíneo adequado ao miocárdio por meio da abordagem das artérias coronarianas. Sendo a RM uma das mais frequentes cirurgias realizadas no mundo, estima-se que o tratamento cirúrgico da DAC se constitui um dos assuntos mais estudados dentre todas as especialidades médicas.

Os objetivos do tratamento cirúrgico são aliviar sintomas, prevenir o IAM, proteger o miocárdio isquêmico, melhorar a função ventricular e prolongar a vida e a sua qualidade. Ainda nos dias de hoje, a RM permanece como uma excelente opção terapêutica para tratamento da DAC, mesmo em pacientes diabéticos, idosos e naqueles com baixa fração de ejeção de ventrículo esquerdo (DALLAN AO e JANETE FB, 2013; MATTOS LA, et al., 2008).

É indiscutível que a RM revolucionou o tratamento das DAC, o que aumentou a sobrevida dos pacientes. Contudo, por ser um procedimento invasivo, com alto risco cirúrgico e morbidade pós-operatória associados, a prevenção da DAC torna-se o melhor caminho para os pacientes com um alto risco cardiovascular e toda a população em geral, visto que, os fatores de risco são, em sua maioria, comportamentais, ou seja, modificáveis. (DALLAN AO e JANETE FB, 2013; MATTOS LA, et al., 2008).

Considerando isto, este trabalho objetiva identificar os fatores de risco prevalentes para o desenvolvimento de DAC em pacientes submetidos a RM, a fim de identificar ações preventivas nos portadores de DAC, o que diminui a necessidade de realização de procedimentos de alta complexidade e garante menor exposição aos riscos decorrentes de procedimentos invasivos.

\section{MÉTODOS}

Trata-se de um estudo retrospectivo, com coleta das variáveis que identificam os fatores de risco para o desenvolvimento de DAC, a partir de análise de prontuários de pacientes submetidos a RM, no período de agosto de 2016 a agosto de 2018, em um hospital público, de uma cidade no interior do estado de São Paulo.

A coleta de dados dos prontuários foi realizada no mesmo hospital, por estudantes de medicina, de acordo com a disponibilidade de horários deles. Obteve-se autorização para realização da pesquisa pelo comitê de ética e pesquisa da Universidade de Franca (UNIFRAN) ( $n^{\circ}$ 2.356.952, CAAE 78965617.1.0000.54.95), de acordo com o Conselho Nacional de Saúde, resolução 466/12, que visa regulamentar pesquisa envolvendo seres humanos e sob a autorização da direção clínica do hospital para acesso aos prontuários.

Para caracterização sociodemográfica foram registrados: gênero, idade, situação conjugal. Para análise de prevalência dos fatores de risco para DAC, identificou-se nos prontuários: peso, idade altura, para cálculo do índice de massa corpórea e sua classificação, hábitos de fumo e consumo de álcool, portadores de HAS, DM e DLP.

Utilizou-se prontuários de pacientes com idade maior a 18 anos, submetidos à $\mathrm{RM}$, no período do ano de 2010 a 2016. Foram excluídos prontuários inelegíveis e os que referiam aos pacientes submetidos a cirurgias valvulares. Para análise de dados, inicialmente armazenou-se todas as informações obtidas durante a coleta de dados de cada campanha no software Microsoft Excel. Em seguida, foram transferidas para o programa estatístico STATA 9.0. Foram apresentadas as variáveis referentes às patologias adquiridas em frequência absoluta e relativa. As variáveis quantitativas: faixa etária, peso, estatura, índice de massa corpórea foram descritos através de média e desvio padrão. 


\section{RESULTADOS}

Ao todo, 206 prontuários foram analisados, sendo 60 (29,1\%) do sexo feminino e 146 (70,8\%) do sexo masculino, com idade média de 65,5 $\pm 8,45$ e 63,5 $\pm 9,16$ respectivamente. A idade mínima foi de 41 anos e máxima de 83 anos, com mediana de 64 anos e média de 64,1 anos (em ambos sexos). 0 peso variou entre $41,4 \mathrm{~kg}$ e $132,15 \mathrm{~kg}$, sua mediana 76,6 kg e média 77,57 kg. O IMC variou entre 18,4 e 42,7 sendo a mediana 28,19 e média 28,6 , como pode ser observado (Tabela 1 ).

Tabela 1 - Distribuição dos pacientes submetidos à cirurgia de revascularização miocárdica segundo características antropométricas. Franca, 2016 a 2018 ( $n=206)$.

\begin{tabular}{cccccc}
\hline Variáveis & Mínimo & Máximo & Mediana & Média & $\begin{array}{c}\text { Desvio } \\
\text { Padrão }\end{array}$ \\
\hline Idade (anos) & 41 & 83 & 64 & 64,10 & 8,98 \\
Peso (Kg) & 41,4 & 132,15 & 76,6 & 77,57 & 14,58 \\
Altura (cm) & 1,42 & 1,86 & 1,65 & 1,64 & 0,09 \\
IMC (Kg/cm²) & 18,50 & 42,70 & 28,19 & 28,60 & 4,26 \\
\hline
\end{tabular}

Legenda: $\mathrm{Kg}$ = quilograma; $\mathrm{cm}$ = centímetro; IMC = Índice de Massa Corpórea.

Fonte: Tavares MMG, et al., 2018.

A (Tabela 2) apresenta a distribuição dos dados dos pacientes analisados, segundo idade, estado civil e fatores de risco prevalentes para o desenvolvimento de DAC. A faixa etária prevalente do total dos pacientes analisados foi de 61 a 70 anos (33,0\%). Os pacientes, em sua maioria, eram casados, com maior prevalência no sexo masculino $101(73,1 \%)$ quando comparado ao sexo feminino $27(50 \%)$. Observa-se que estas são mais submetidas à RM principalmente após aos 60 anos de idade, enquanto que, no sexo masculino, observou-se um padrão ocorrendo com maior frequência dos 41 aos 70 anos de idade.

A grande maioria dos prontuários pertenciam a pacientes que são ou já foram casados, totalizando 173 $(90,2 \%)$, sendo destes $128(66,6 \%)$ casados, $26(13,5 \%)$ divorciados, $19(9,8 \%)$ viúvos e $19(9,8 \%)$ solteiros. Quanto à distinção entre os IMC, observou-se que houve maior prevalência entre pacientes com sobrepeso totalizando 74 pacientes (46,8\%), seguido por obesidade grau 146 pacientes $(29,1 \%)$, faixa de peso ideal com 28 indivíduos (17,7\%), obesidade grau 2 com 7 indivíduos (4,4\%) e grau 3 com 3 pacientes $(1,8 \%)$.

Ao todo, foram identificados 62 tabagistas $(30 \%)$, com maior proporção entre a população masculina $(32,8 \%)$ que a feminina (23,3\%). O etilismo foi fator encontrado em apenas $(7,2 \%)$ do espaço amostral analisado, sendo um pouco mais evidente nas mulheres $(8,3 \%)$ contra $(6,8 \%)$ relatado pelos homens no prontuário.

HAS foi observada em 172 pacientes (83,4\%), sendo mais evidente em $85 \%$ das mulheres e em $82,8 \%$ homens. DM também foi evidente totalizando $50,4 \%$ dos indivíduos, sendo formado por $56,6 \%$ mulheres e 47,9\% homens. Encontrou-se DLP em 19,4\% dos prontuários analisados, sendo 20,5\% homens e 16,6\% mulheres.

Os principais fatores de risco para o desenvolvimento de DAC identificados foram: classificação do IMC, que apresentou grande percentual na categoria "Sobrepeso" 74 (46,8\%) seguido por "Obesidade grau I" 46 $(29,1 \%)$, e a HAS presente em $172(83,4 \%)$ dos pacientes analisados. Respectivamente, outros fatores de risco prevalentes identificados foram: DM 104 (50,4\%), Tabagismo 62 (30\%), DLP 40 (19,4\%) e Etilismo 15 $(7,2 \%)$. 
Tabela 2 - Distribuição dos pacientes submetidos à RM segundo idade, estado civil e fatores de risco prevalentes para o desenvolvimento de DAC. Franca, 2016 a $2018(\mathrm{n}=206)$.

\begin{tabular}{|c|c|c|c|c|c|c|}
\hline \multirow{3}{*}{ Variáveis } & \multicolumn{4}{|c|}{ Sexo } & & \\
\hline & \multicolumn{2}{|c|}{ Feminino } & \multicolumn{2}{|c|}{ Masculino } & \multicolumn{2}{|c|}{ Total } \\
\hline & $n$ & $\%$ & $\mathrm{n}$ & $\%$ & $\mathbf{n}$ & $\%$ \\
\hline \multicolumn{7}{|l|}{ Faixa etária (anos) } \\
\hline $41-50$ & 3 & 5 & 13 & 8,9 & 16 & 7,7 \\
\hline $51-60$ & 16 & 26,6 & 47 & 32,1 & 63 & 30,5 \\
\hline $61-70$ & 21 & 35,0 & 47 & 32,1 & 68 & 33,0 \\
\hline $71-80$ & 20 & 33,3 & 35 & 23,9 & 55 & 26,6 \\
\hline$>80$ & 0 & 0 & 4 & 2,7 & 4 & 1,9 \\
\hline \multicolumn{7}{|l|}{ Estado Civil } \\
\hline Solteiro(a) & 7 & 12,9 & 12 & 8,6 & 19 & 9,8 \\
\hline Casado(a) & 27 & 50 & 101 & 73,1 & 128 & 66,6 \\
\hline Divorciado(a) & 7 & 12,9 & 19 & 13,7 & 26 & 13,5 \\
\hline Viúvo(a) & 13 & 24 & 6 & 4,3 & 19 & 9,8 \\
\hline \multicolumn{7}{|c|}{ Classificação do IMC (kg/cm²) } \\
\hline Baixo Peso & 0 & 0 & 0 & 0 & 0 & 0 \\
\hline Peso ideal & 11 & 25,5 & 17 & 14,7 & 28 & 17,7 \\
\hline Sobrepeso & 16 & 37,2 & 58 & 50,4 & 74 & 46,8 \\
\hline Obesidade grau I & 12 & 27,9 & 34 & 29,5 & 46 & 29,1 \\
\hline Obesidade grau II & 3 & 6,9 & 4 & 3,4 & 7 & 4,4 \\
\hline Obesidade grau III & 1 & 2,3 & 2 & 1,7 & 3 & 1,8 \\
\hline \multicolumn{7}{|l|}{ Tabagista } \\
\hline Sim & 14 & 23,3 & 48 & 32,8 & 62 & 30 \\
\hline Não & 46 & 76,6 & 98 & 67,1 & 144 & 69,9 \\
\hline \multicolumn{7}{|l|}{ Etilista } \\
\hline Sim & 5 & 8,3 & 10 & 6,8 & 15 & 7,2 \\
\hline Não & 55 & 91,6 & 136 & 93,1 & 191 & 92,7 \\
\hline \multicolumn{7}{|l|}{ Hipertenso(a) } \\
\hline Sim & 51 & 85 & 121 & 82,8 & 172 & 83,4 \\
\hline Não & 9 & 15 & 25 & 17,1 & 34 & 16,5 \\
\hline \multicolumn{7}{|l|}{ Diabético(a) } \\
\hline Sim & 34 & 56,6 & 70 & 47,9 & 104 & 50,4 \\
\hline Não & 26 & 43,3 & 76 & 52,0 & 102 & 49,5 \\
\hline \multicolumn{7}{|l|}{ Dislipidêmico } \\
\hline Sim & 10 & 16,6 & 30 & 20,5 & 40 & 19,4 \\
\hline Não & 50 & 83,3 & 116 & 79,4 & 166 & 80,5 \\
\hline
\end{tabular}

Legenda: $\mathrm{Kg}$ = quilograma; $\mathrm{cm}$ = centímetro; IMC = Índice de Massa Corpórea.

Fonte: Tavares MMG, et al., 2018.

\section{DISCUSSÃO}

Este estudo visou identificar a prevalência de fatores de risco para desenvolvimento de DAC nos pacientes submetidos a RM, tais como HAS, excesso de peso, DM, sexo, idade, tabagismo, etilismo, DLP e estado civil que serão abordados separadamente nos próximos parágrafos. 


\section{Hipertensão}

Dentre os fatores abordados, a HAS foi encontrada em $83,4 \%$ dos pacientes neste estudo, sendo o fator de risco mais prevalente neste espaço amostral. Com exceção de um artigo encontrado (FEIJO MKEF, et al., 2010), todos os outros que foram selecionados para a discussão trouxeram a HAS como fator mais prevalente para desenvolvimento tanto de DAC quanto de SCA e seus desfechos, sendo que, na exceção, a HAS é o segundo fator de risco mais prevalente (LIMA FET, et al., 2004; SILVA BAG e LEITE MAR, 2010; BRUNORI et al., 2014; BARBOSA JL, et al., 2018).

A HAS é um dos fatores de riscos mais importantes para o desenvolvimento de doença aterosclerótica, sendo, na metade das vezes, a causa das mortes por DAC no Brasil (MALACHIAS MVB et al., 2016). Devese ao fato de que o sangue, ao chegar em alta pressão nos capilares sanguíneos, causa disfunção endotelial, levando a uma redução dos tônus vasculares ou a uma redução da capacidade de vasodilatação, o que tem sido documentado em quase todas as condições associadas à aterosclerose e à DAC no Brasil e no mundo (TOLEDO JCY, et al., 2015).

Apesar disso, a taxa de mortalidade tem demonstrado uma tendência à redução desde 2010, quando comparada com o período de 2002 a 2009, o que se deve possivelmente a maior quantidade de diagnósticos ou ao tratamento otimizado da doença (MALACHIAS MVB, et al., 2016). Deve-se, portanto, intervir nesses pacientes precocemente, identificando seus níveis pressóricos e tratando-os de acordo com as novas diretrizes, a fim de diminuir os possíveis efeitos deste fator de risco.

\section{Excesso de Peso}

Observou-se, neste estudo, que $82,1 \%$ dos pacientes submetidos à RM estavam com o IMC elevado e foram classificados principalmente como portadores de sobrepeso e obesidade grau I, apesar de ser considerado um fator de risco com baixa especificidade (GOMES F, et al., 2010), o IMC é considerado um dos principais fatores de risco para SCA (FEIJÓ MKEF, et al., 2010).

Observaram que, quanto maior o IMC, maior o risco para o desenvolvimento de DAC. Há também íntima relação do aumento do IMC e a mortalidade, principalmente naqueles que possuem obesidade abdominal. $O$ fato de termos encontrado mais indivíduos com sobrepeso e obesidade 1 pode evidenciar que, talvez, pacientes mais obesos venham a falecer antes de serem submetidos à RM, entretanto mais estudos são necessários para elucidar tal hipótese (GONZALEZ AB, et al., 2010).

Em pessoas do sexo feminino, a obesidade encontra-se duas vezes mais evidente nos pacientes com DAC (BRUNORI EHFR, et al., 2014; SILVA BAG e LEITE MAR, 2010). No atual estudo, encontrou-se uma prevalência menor de mulheres (60 indivíduos) que realizaram RM em relação ao sexo oposto (206 indivíduos), o que pode corroborar com o aumento da mortalidade relacionado à obesidade, que ocorreria previamente ao procedimento por causas cardiovasculares ou quaisquer outras. Apesar de a obesidade ser um fator de risco independente para DAC, deve-se considerar também que as combinações de fatores de risco são determinantes para o aumento da incidência de eventos cardiovasculares, e isto pode se relacionar à RM (FEIJÓ MKEF, et al., 2010; PIEGAS LS, et al., 2015).

\section{Diabetes Mellitus}

A DM é evidenciada, neste estudo, como segundo fator de risco mais prevalente, $50,4 \%$, próximo à literatura que traz $46,2 \%$ de prevalência (LIMA FET, et al., 2012). Em outros estudos evidencia-se dados um pouco mais baixos com variação entre 27\% e 34\% (BRUNORI EHFR, et al., 2014; FEIJÓ MKEF, et al., 2010; SILVA BAG e LEITE MAR, 2010). A importância da inclusão de pacientes diabéticos neste estudo se deve ao fato de que essa patologia está presente significativamente em pacientes com DAC, uma vez que, pacientes hiperglicêmicos possuem complicações micro e macro vasculares devido aos danos teciduais causados pelas macromoléculas de glicose endotélio capilar (PEREIRA JAC e SANTOS LC, 2012)

\section{Sexo}

Neste trabalho, identificou-se a prevalência do sexo masculino (73,1\%). Apesar disso, evidencia-se maior incidência de IAM nas mulheres, estando estas provavelmente mais expostas à letalidade por IAM, 
considerando um estudo realizado (FREITAS DMO, et al., 2018), em que constataram que as mulheres possuem mais propensão para o desenvolvimento de IAM, provavelmente por possuírem artérias menos calibrosas que os homens e, com o avançar da idade, chegando à menopausa, o estrógeno encontra-se reduzido, que ajuda a evitar o acúmulo de lipoproteína de baixa densidade (LDL), e há o aumento da lipoproteína de alta densidade (HDL), ou seja, talvez as mulheres podem chegar a óbito antes mesmo de serem submetidas à RM por estarem expostas a um risco maior. Apesar de tal hipótese ser aventada, mais estudos seriam necessários para chegar a uma conclusão.

\section{Idade}

Naqueles pacientes submetidos à RM, encontrou-se dados que se assemelham a outros trabalhos, ocorrendo em $61,5 \%$ dos pacientes acima de 61 anos de idade neste e em $68 \%$ naqueles com mais de 60 anos de idade na literatura. Com o avançar da idade, algumas das comorbidades se fazem mais presentes, como é o caso da HAS, o que propicia o surgimento de DAC. Além disso, o paciente senil apresenta uma maior fragilidade vascular, propiciando o surgimento de lesões endoteliais, o que justifica a maior prevalência de DAC e RM em pacientes idosos (SCHEER C e RIBEIRO JP, 2009; MASSA KHC, et al., 2019).

\section{Tabagismo}

Neste trabalho, encontrou-se prevalência de $30 \%$ de pacientes tabagistas, em outros estudos encontrouse dados semelhantes, como um trabalho realizado em Minas-Gerais, em que 37\% dos pacientes com SCA são tabagistas (SILVA BAG e LEITE MAR, 2010).

O risco de morte súbita por doença cardíaca é 10 vezes maior em pacientes fumantes, principalmente os que fumam mais de 20 cigarros por dia (COLOMA M, 2014). Isso porque as propriedades do cigarro atuam diminuindo a elasticidade arterial, além de atuarem diretamente no endotélio capilar, levando a redução de oxido nítrico, tornando as artérias mais suscetíveis às mais diversas formas de agressão endotelial (MUNIZ LC, et al., 2012; COLOMA M, 2014).

\section{Etilismo}

Neste estudo, encontrou-se prevalência de $7,2 \%$ de alcoolismo durante análise dos prontuários. $O$ etilismo não recebeu muito enfoque das literaturas encontradas, sendo apenas citado como um fator de risco proposto pela OMS e por outros estudos publicados (BRUNORI EHFR, et al., 2014; FEIJÓ MKEF, et al., 2010; LIMA FET, et al., 2012), isso pode ocorrer pois não se sabe definitivamente a relação entre etilismo e DAC (FREITAS DMO, et al., 2018) citam-no como um fator relacionado ao estilo de vida, que possui influência no mecanismo fisiopatológico da DAC, consequentemente do IAM e da RM. Um estudo realizado com 6632 pacientes demonstrou que o etilismo de leve a moderado não se apresentou como risco para desenvolvimento de isquemia durante a realização de ecocardiografia sob estresse físico (FONTES VJB, et al., 2017).

Observou-se que o consumo de álcool aumentaria os níveis de HDL, entretanto não está indicado para pacientes que possuam hipertrigliceridemia, pois sua ingestão excessiva pode levar à piora desta condição, sendo esta considerada um importante fator de risco para DAC (FALUDI AA, et al., 2017).

\section{Dislipidemia}

A literatura traz DLP como fator de risco encontrada entre 40\% a 76,6\% dos casos (BRUNORI EHFR, et al., 2014; FEIJÓ MKEF, et al., 2010; LIMA FET, et al., 2012; SILVA BAG e LEITE MAR, 2010; BARBOSA JL, et al., 2018). Neste trabalho, ocorreu em apenas $19,2 \%$, o que se mostra divergente da literatura, apesar de que, neste trabalho, nenhum prontuário diferenciou o tipo de DLP, ou seja, dislipidemia pura, mista, hipertrigliceridemia isolada etc. Isso pode ocorrer devido a um possível subdiagnóstico da doença, ao paciente não referir que possui DLP ou, ainda, ao fato de o profissional de saúde não anotar em prontuário, o que pode subestimar a prevalência de tal patologia no paciente submetido a RM.

A aterosclerose é uma doença inflamatória crônica de origem multifatorial, que ocorre em resposta à agressão ao endotélio. Sua formação inicia-se com agressão ao endotélio vascular, propiciando a adesão das lipoproteínas na parede arterial, levando a formação de placa e sua possível ruptura, a depender de outros fatores associados (XAVIER HT, et al., 2013). Ao compreendermos a fisiopatologia da DLP na DAC, 
entendemos a importância do diagnóstico e tratamento adequado desta, visando a metas de LDL adequadas para cada paciente.

\section{Estado Civil}

Neste trabalho, evidenciou-se maior prevalência de RM em pessoas casadas divorciadas ou viúvas, isso pode ser decorrente de que neste estudo o espaço amostral corresponde a pacientes com idade acima de 41 anos, sendo este um possível momento da vida em que as pessoas, em sua maioria, encontram-se nos estados civis supracitados. Apesar disso, um trabalho realizado por (ZANCHET AT e MARIN AH, 2014) apontou que pessoas casadas e divorciadas estão mais propensas ao desenvolvimento de IAM.

\section{Propostas de intervenção}

Com esses dados, a adoção de alimentação saudável, assim como a prática de atividade física regular são importantes para a tentativa de diminuir os fatores de risco para desenvolvimento de DAC e consequentemente os possíveis fatores de risco encontrados neste trabalho associados à $\mathrm{RM}$. $\mathrm{O}$ paciente deve melhorar seu IMC, cessar tabagismo, controlar a pressão arterial, DM e DLP (PIEGAS LS, et al., 2015).

\section{CONCLUSÃO}

Os principais fatores de risco para DAC em pacientes submetidos à RM foram: HAS, seguido por pacientes idosos (principalmente maiores de 64 anos), com sobrepeso, tabagistas, dislipidêmicos e por último, etilistas. Apesar de serem escassas as referências que relacionam o estado civil com a DAC, observou-se uma alta prevalência de indivíduos que são ou já foram casados que foram submetidos à RM. Apesar disso, muitos desses fatores podem ser evitados, a adoção de medidas precoces para o tratamento dos fatores modificáveis é necessária, podendo alterar o curso da doença, evitando que o paciente seja submetido à RM. Medidas socioeducativas podem ser tomadas ou intensificadas para a prevenção da SCA e, consequentemente da RM, como a mudança no estilo de vida. A literatura disponível de forma gratuita que correlaciona os fatores de risco para DAC com RM é escassa, sendo necessários mais estudos.

\section{AGRADECIMENTOS E FINANCIAMENTO}

Agradecemos ao Dr. Edson Alves Margarido e todos os funcionários da sala de prontuário, os quais foram fundamentais para a realização deste trabalho.

\section{REFERÊNCIAS}

1. ALVES A, MARQUES IR. Fatores relacionados ao risco de Doença Arterial Coronariana entre estudantes de enfermagem. Rev Bras Enferm, Brasília, 2009; 62(6): 883-888.

2. BARBOSA JL, et al. Impacto dos Fatores de Risco para Doença Arterial Coronariana nos Gastos Hospitalares dos Pacientes Submetidos à Cirurgia de Revascularização do Miocárdio no SUS, 2018; 31(2): 90-96.

3. BRUNORI EHFR, et al. Association of cardiovascular risk factors with the different presentations of acute coronary syndrome. Rev Lat Am Enfermagem, 2014; 22(4): 538-46.

4. COLOMA M. Tabagismo e doença coronariana. In: Secretaria Municipal de Administração. Rio de Janeiro, 2014. Disponível em http://www.rio.rj.gov.br/dlstatic/10112/4713572/4132009/diamundialdocoracao.pdf.

5. DALLAN LAO, JATENE FB. Revascularização miocárdica no século XXI. Rev Bras Cir Cardiovasc, 2013; 28(1): 137144.

6. FALUDI AA, et al. Atualização da diretriz brasileira de dislipidemias e prevenção da aterosclerose - 2017. Arq Bras Cardiol, 2017; 109(1): 1-76.

7. FEIJÓ MKEF, et al. Fatores de risco para doença arterial coronariana em pacientes admitidos em unidade de hemodinâmica. Rev Gaúcha Enferm, 2010; 30(4): 641-647.

8. FERNANDES PM. Estado atual da cirurgia de revascularização do miocárdio. Rev Med, São Paulo, 2008; 87(2): 9298.

9. FERREIRA AG, et al. A doença arterial coronariana e o envelhecimento populacional: como enfrentar esse desafio? Revista HUPE, 2013; 12(1): 13-24.

10. FONTES VJB, et al. Consumo leve e moderado de álcool e isquemia miocárdica à ecocardiografia sob estresse físico. Int. J. Cardiovasc. sci., 2018; 31(3).

11. FREITAS DMO, et al. Síndrome coronariana aguda: Parte 2 - Fatores de risco e Tratamento. REAS, 2018; 15: 19351941. 
12. GONZALEZ AB, et al. Body-Mass Index and Mortality among 1.46 Million White Adults. N Engl J Med, 2010; 363(23): 2211-2219.

13. GOMES F, et al. Obesidade e doença arterial coronariana: papel da inflamação vascular. Arq. Bras. Cardiol, 2010; 94(2): 273-279.

14. LIMA FET, et al. Fatores de risco da doença coronariana em pacientes que realizaram revascularização miocárdica. Rev Rene, 2012; 13(4): 853-860.

15. LIMA RC, et al. Diretrizes da cirurgia de revascularização miocárdica valvopatias e doenças da aorta. Arq Bras Cardiol, 2004; 82(5): $20 \mathrm{p}$.

16. MALACHIAS, MVB et al. $7^{\underline{a}}$ Diretriz Brasileira de Hipertensão Arterial. Arquivos Brasileiros de Cardiologia, 2016; 107(3): $83 \mathrm{p}$.

17. MASSA, KHC, et al. Análise da prevalência de doenças cardiovasculares e fatores associados em idosos, 20002010. Ciênc. saúde coletiva, 2019; 4(1): 105-114.

18. MATTOS LA, et al. Diretrizes da Sociedade Brasileira de Cardiologia - Intervenção Coronária Percutânea e Métodos Adjuntos Diagnósticos em Cardiologia Intervencionista. Rev Bras Cardiol Invas, 2008; 109(1).

19. MUNIZ LC, et al. Fatores de riscos comportamentais acumulados para doenças cardiovasculares no sul do Brasil. Rev Saúde Pública, 2012; 36(3): 534-542.

20. PEREIRA JAC, SANTOS LC. Diabetes Mellitus e doença cardiovascular. Tese (Mestrado Integrado em Medicina). Faculdade de Medicina da Universidade de Coimbra, 2012: 55p.

21. PIEGAS LS, et al. V Diretriz da Sociedade Brasileira de Cardiologia sobre Tratamento do Infarto Agudo do Miocárdio com Supradesnível do Segmento ST. Arq Bras Cardiol, 2015; 105(2).

22. SCHEER C, et al. Gênero, idade, nível social e fatores de risco cardiovasculares: considerações sobre a realidade brasileira. Arq. Bras. Cardiol, 2009; 93(3): 54-56.

23. Silva BAG, LEITE MAR. Fatores de risco para síndrome coronariana aguda em pacientes internados na terapia intensiva de um hospital em Contagem, MG TT - Risk factors for acute coronary syndrome in patients hospitalized in intensive care at a hospital in Contagem, Brazil. Rev méd Minas Gerais, 2010; 20(3): 10-6.

24. TOLEDO JCY, et al. Disfunção endoletial e hipertensão arterial. Rev. bras. hipertens, 2015; 22(3): 84-92.

25. XAVIER HT, et al. V diretriz brasileira de dislipidemias e prevenção de aterosclerose. Arq. Bras. Cardiol, $2013 ; 101(4)$.

26. ZANCHET AT, MARIN AH. Perfil psicossocial de pacientes com syndrome coronariana aguda. Psic, Saúde \& Doenças. Lisboa, 2014; 15(3): 656-770. 